\title{
Heterogeneidad en el impacto de la política de becas en la escolaridad secundaria postobligatoria en España: un análisis por subgrupos poblacionales*
}

\author{
Heterogeneity of the impact of scholarships in Spain in the upper \\ secondary education: a sub-population analysis
}

Mauro Mediavilla**

\begin{abstract}
Resumen
El presente trabajo analiza el impacto de las becas sobre la probabilidad de finalizar con éxito el nivel secundario postobligatorio en España en el caso de diferentes subgrupos poblacionales. Se aplica un diseño cuasiexperimental (Propensity score matching) a partir de la Encuesta de Condiciones de Vida (ECV-2006). Los resultados confirman la existencia de efectos diferenciales positivos (con relación al impacto medio) de la política educativa en el caso de las mujeres y de los alumnos provenientes de hogares desfavorecidos, demostrando la existencia de heterogeneidad en el impacto de la política analizada.
\end{abstract}

Palabras clave: Becas; Educación secundaria postobligatoria; Propensity Score Matching; Impactos diferenciales; ECV-06.

Clasificación JEL: J38; I21; C21.

\begin{abstract}
The main objective of this study is to identify and quantify the existence of an impact on upper secondary completion rates of a scholarships program applied to different sub-populations in Spain. The evaluation is performed applying an ex-post quasi-experimental approach technique, the propensity score matching, to microdata obtained from the European Union Statistics on Income and Living Conditions (EU-SILC 2006). Results confirm the existence of a differential positive
\end{abstract}

* Este trabajo ha contado con el apoyo financiero de la Fundació Bofill (Barcelona, España).

** Universitat de València \& IEB \& GIPE, Departament d'Economia Aplicada, Avenida Tarongers, s.n. 46022 Valencia (España).Teléfono: 963828634.E-mail: Mauro.Mediavilla@ uv.es.

Agradezco los valiosos comentarios y sugerencias recibidos por parte del evaluador y del editor. 
effect of scholarships for women and students from disadvantaged households, demonstrating the existence of heterogeneous impacts of the policy analysed.

Key words: Scholarships; Upper secondary education; Propensity Score Matching; Differential impacts; EU-SILC-06.

Classification JEL: J38; I21; C21.

\section{INTRODUCCIÓN}

Uno de los principales elementos que ha centrado el debate en los últimos años, con relación al nivel secundario postobligatorio en España, es la gran cantidad de individuos que no continúan sus estudios más allá de la escolarización obligatoria y que se cuantifica mediante una alta tasa de abandono escolar prematuro $^{1}$. La tasa del $28,4 \%$ en 2010 se encuentra muy por encima de la media europea de $27 \%(14,1 \%)$ y del objetivo comunitario para 2020 que se sitúa en el 10\% (European Commission, 2010). Asimismo, resulta preocupante el notorio estancamiento observado para este indicador que en 2005 era de $30,8 \%{ }^{2}$.

Esta problemática, tratada en España desde diferentes enfoques como las aproximaciones cualitativas (Adame Obrador y Salvà Mut, 2010), las técnicas de regresión (Alegre Canosa y Benito Pérez, 2010) y las reflexiones globales (Escudero et al., 2009; Roca Cobo, 2010), presenta consecuencias negativas para el individuo. En líneas generales, un bajo nivel educativo alcanzado constituye una evidente desventaja para la incorporación en el mercado de trabajo dentro de una sociedad cada vez más basada en el conocimiento, y se traduce en menores tasas de empleo, mayores tasas de desempleo y una relativa menor retribución salarial. Asimismo, a mediano y largo plazo, la ausencia de un adecuado nivel educativo podría potenciar el riesgo de exclusión social y las tasas de criminalidad e, incluso, incrementar los problemas de salud (Rumberger, 1987; Katz y Murphy, 1992; OCDE, 1998; GHK, 2005).

Con el objeto de paliar este problema el gobierno español, en los últimos años, ha aplicado diferentes estrategias destinadas a mejorar la oferta y potenciar la demanda educativa. Se ha desarrollado una política de flexibilización de los horarios escolares, de reducción del ratio alumno/profesor, de creación de planes de estudio focalizados y se han llevado a cabo cambios en el sistema educativo con el objeto de permitir a los alumnos, que han salido de los mismos sin la suficiente preparación para poder integrarse adecuadamente en el mercado laboral, puedan reinsertarse mediante una "segunda oportunidad educativa" 3 . A

1 Abandono escolar prematuro: porcentaje de jóvenes de 18 a 24 años que han completado como máximo la primera etapa de la educación secundaria y no continúan ningún estudio o formación adicional.

2 Comparaciones con años anteriores no son posibles debido a la implementación tardía de conceptos y definiciones armonizadas en el ámbito de la Unión Europea.

3 Programa de Capacitación Profesional Inicial, Programa de Escuelas Taller, Casas de Oficio y Talleres de Empleo; Programa de Educación Permanente para Adultos (EPA) y, finalmente, las enseñanzas secundarias a distancia. 
su vez, se ha ido ampliando y consolidando una política de becas con el objetivo, en este caso específico, de disminuir las diferencias económicas de partida. En este sentido, la intervención del Estado queda fundamentada a partir de las conclusiones de diversos modelos teóricos que revelan las consecuencias no deseadas de las imperfecciones del mercado de capitales relacionadas con la asimetría informativa. Las mismas provocan la no aceptación del capital humano esperado como garantía para el financiamiento de la formación del individuo (Barham et al., 1995; Berti Ceroni, 2001).

En tal contexto, el presente estudio tiene como principal objetivo analizar la relación existente entre las políticas públicas educativas y la deserción escolar. Se pretende estudiar, en concreto, el papel de las becas y ayudas al estudio en la probabilidad de finalizar con éxito el nivel secundario postobligatorio y, específicamente, se intenta determinar y cuantificar la existencia de un impacto de las políticas de becas, llevadas a cabo en España durante el período 2004-2005, en las probabilidades de concluir este nivel educativo según diferentes subgrupos poblacionales.

En cuanto a la metodología, se aplicará una evaluación de impacto (ex post), con información individual, para conocer si hubo cambios en el nivel educativo de la población analizada y si los mismos son atribuibles a la intervención. Concretamente, el análisis se realiza mediante un diseño cuasiexperimental a partir de la aplicación del Propensity score matching. Para realizar el análisis se emplea la Encuesta de Condiciones de Vida (ECV), publicada por EUROSTAT en 2009, que brinda información longitudinal de los individuos y, por tanto, permite realizar el seguimiento de los mismos mediante su paso por el nivel educativo analizado. Los resultados obtenidos indican la existencia de efectos diferenciales de las becas por subgrupos poblacionales. Se concluye que ciertos colectivos tienden a rentabilizar, en términos educativos, la ayuda recibida en mayor medida que otros grupos poblacionales. Es el caso de las mujeres y aquellos pertenecientes a hogares con un nivel de renta relativamente bajo.

El presente trabajo se inicia con una breve revisión de la literatura con relación al impacto de la política de becas en el rendimiento educativo (capítulo 2) y una sucinta descripción de la situación de las becas en España (capítulo 3). En el capítulo 4 se expone la metodología utilizada y, en el capítulo 5, se realiza un análisis descriptivo de la base de datos y las variables empleadas en las estimaciones. En el capítulo 6 se presentan los resultados más destacados, un análisis de la calidad de los emparejamientos y una evaluación agregada del impacto; por último, en el capítulo 7 se plantean las principales conclusiones.

\section{Antecedentes}

Existe en la literatura una serie de desarrollos teóricos y aplicaciones empíricas que se han elaborado con el objeto de explicar las diferencias en los rendimientos académicos observados en los individuos mediante el análisis de una serie de factores que, previsiblemente, impactarían en las posibilidades educativas reales de los alumnos.

Con el objetivo de clarificar la exposición de los diferentes factores mencionados en la literatura, y a partir de la sistematización conceptual planteada por Dronkers (2008), se divide la enumeración de los mismos en cinco categorías: 
la del propio individuo, de sus progenitores, del hogar donde reside, de la escuela donde asiste y del entorno global que lo rodea. Este apartado centrará su atención en el último ámbito mencionado, ya que aquí es donde se mencionan los efectos de las políticas públicas en el rendimiento educativo.

En este marco se introduce la potencial acción del Estado mediante diferentes políticas públicas, como, por ejemplo, las ayudas directas (en forma de becas y ayudas al estudio u otro tipo de transferencia) y las prestaciones fiscales. A nivel empírico, y mediante diferentes aproximaciones referidas a la educación superior, los estudios analizados demuestran que existe un efecto positivo de las ayudas públicas, vía becas o préstamos subvencionados, sobre las tasas de escolarización o de graduación (McPherson y Schapiro, 1991; Wetzel et al., 1999; DesJardins et al., 2002; Deke, 2003; Kim, 2007; Monks, 2009).

En el caso español, Marcenario Gutiérrez y Navarro Gómez (2001), a partir de una aproximación multivariante (modelo probit), intentan determinar los condicionantes de la demanda de educación superior. Basados en el Panel de Hogares de la Unión Europea (PHOGUE) encuentran que haber sido becario en la enseñanza secundaria tiene un marcado impacto sobre la decisión del estudiante de continuar sus estudios superiores. A su vez, De Pablos Escobar y Gil Izquierdo (2008) emplean un modelo de comportamiento mediante una aproximación multivariante (probit) para determinar los factores que inciden en la demanda de educación universitaria en España. A partir de la Encuesta de Condiciones de Vida (2004) detectan que las becas tienen un efecto reducido pero significativo en las probabilidades de que el individuo se encuentre escolarizado en el nivel superior.

En cuanto a los antecedentes empíricos referidos, específicamente a los efectos de las ayudas públicas sobre la deserción escolar en los niveles anteriores a la educación superior, a continuación se describen, brevemente, cuatro estudios que permiten observar diferentes aproximaciones empleadas en la literatura empírica.

En primer lugar, Skoufias (2001) analiza uno de los mayores programas sociales destinados a los hogares más desfavorecidos en México llamado PROGRESA (Programa de Educación, Salud y Alimentación). El programa, dirigido a los hogares rurales en siete estados, consiste en transferencias en dinero a las familias (específicamente, a las madres) en el caso que el alumno se encuentre matriculado y asista regularmente al centro escolar. Su objetivo es la mejora en las tasas de matrículas, de asistencia al centro escolar y de logro educativo de los individuos beneficiarios. El sistema de ayuda se basa en becas dirigidas a los estudiantes (con objeto de cubrir los costes de escolarización y los costes de oportunidad de no estar en el mercado de trabajo); la mejora de la oferta y la calidad de los servicios educativos y la implantación de medidas que tienen como objeto concienciar a los progenitores sobre las ventajas que tiene la educación para sus hijos. Para evaluar el impacto del programa se emplea un análisis cuasiexperimental de diferencias en diferencias con datos de noviembre de 1997 y noviembre de 1999. El mismo revela un incremento en las tasas de matrículas para los diversos niveles educativos analizados y una caída en las tasas de deserción escolar en el período de transición entre la escolaridad primaria y secundaria.

En segundo lugar, Cardoso y Portela Souza (2004) estudian los efectos de las transferencias en dinero directas a las familias en Brasil, a partir de la apli- 
cación del programa denominado "Bolsa escola", vigente en la segunda mitad de los noventa y dirigido a las familias con miembros entre los 7 y 14 años (correspondientes al ciclo de educación básica). El objetivo del programa era incrementar los logros educativos de los individuos beneficiarios de la ayuda, con el objeto de mejorar las posibilidades futuras de los mismos en cuanto a su inserción en el mercado de trabajo. Se planteaba como requisito para seguir gozando de la prestación un mínimo de asistencia al centro escolar. A partir de un análisis cuasiexperimental (Propensity score matching), encuentran un impacto positivo y significativo de las transferencias en los niveles de escolarización de los alumnos.

En tercer lugar, Cameron (2009) analiza el impacto de un plan de becas realizado en Indonesia con el objetivo de reducir las tasas de deserción escolar en el nivel secundario inferior. Las mismas se diseñaron para cubrir gran parte de los gastos de escolarización, aunque las familias receptoras podían destinar el importe a otros gastos del hogar. Para la estimación del impacto se aplica una aproximación multivariante (probit) donde se incorpora, como variable independiente, una dummy referida a las ayudas recibidas en forma de becas. Los resultados muestran que el programa reduce significativamente la probabilidad de desertar en la escolaridad media.

Por último, y para el caso español, la Agencia de Evaluación y Calidad (AEVAL, 2009) presenta una primera evaluación del sistema general de becas educativas. Su objetivo es valorar en qué medida las becas, y en particular aquellas referidas a la educación postobligatoria (secundaria postobligatorio y superior), está favoreciendo el acceso equitativo a la educación. Se realiza un análisis descriptivo de las becas y el contexto en que las mismas operan. Asimismo, y a partir de dos fuentes de información secundaria (Encuesta de Condiciones de Vida, 2006 y la Encuesta de Transición Educativo-Formativa e Inserción Laboral, 2005), se intenta establecer las causas del abandono escolar prematuro, la eficiencia de las becas y su grado de cobertura. Entre sus resultados más destacados se observa que los principales determinantes del abandono son la desmotivación, los malos resultados y el ingreso al mercado laboral. Asimismo, y a partir de una estimación multivariante (probit) para ambos niveles educativos analizados, se comprueba que los requisitos formales para la concesión de las becas se estarían cumpliendo en la práctica.

En este marco, la contribución central del presente trabajo se basa en cuatro aspectos. En primer lugar, la utilización de un diseño cuasiexperimental que permite aislar el impacto de la política de becas de otras actuaciones que puedan afectar contemporáneamente al individuo y, además, que brinda la posibilidad de determinar la existencia de impactos diferenciales de la política de becas según subgrupos poblacionales. En segundo lugar, la realización de un ejercicio de evaluación de política centrado en el nivel secundario postobligatorio, el cual explica gran parte del abandono escolar prematuro observado en España. En tercer lugar, el empleo de una base de datos reciente que posee información individual y longitudinal de los individuos analizados. La misma permite controlar la estimación por diferentes características no observables que tradicionalmente son omitidas (inteligencia y motivación, por ejemplo) y que producen un claro sesgo en estimaciones realizadas a partir de bases de corte transversal. Finalmente, y debido a que la aplicación de esta metodología a partir de los datos de la Encuesta de Condiciones de Vida (2009) para determinar el efecto neto y diferencial de 
las becas en España no tiene antecedentes en la literatura, el trabajo intenta abrir una nueva vía de investigación con el objetivo de aportar nuevas evidencias a partir de una estimación que mejora la robustez de anteriores aproximaciones.

\section{Las Becas en España: Un Análisis de Situación}

La literatura teórica indica que la educación y el aprendizaje del individuo dependen de una multitud de factores personales y ambientales. Ello implica que las desigualdades existentes en la sociedad podrían llegar a ser reproducidas por el sistema educativo, generando una desigualdad manifiesta entre los ciudadanos en cuanto a las posibilidades reales de educarse y una evidente pérdida de eficiencia en la medida que personas capacitadas se vean imposibilitadas de acceder a niveles educativos superiores. Esta situación fundamenta la intervención pública, que se aplica persiguiendo dos objetivos básicos: la igualdad de oportunidades y la eficiencia. En este sentido, las becas constituyen una herramienta que permite dirigir la acción pública hacia determinadas personas con el objeto de intentar disminuir los desequilibrios de partida.

En el ámbito español las becas y ayudas al estudio ${ }^{4}$ (becas, en adelante) intentan cubrir diversos costes asociados a la escolarización, siendo los mismos: los costes directos (matrícula), indirectos (material escolar, transporte, residencia) y los costes de oportunidad (salarios dejados de percibir al estar estudiando). En la actualidad, su principal objetivo pasa por garantizar la igualdad de oportunidades de acceso a los diferentes niveles educativos y, en líneas generales, el sistema vigente en España no resulta muy diferente a los existentes en otros países desarrollados. Combina las becas de carácter general, que tienen el objetivo prioritario de permitir la continuidad escolar en los niveles no gratuitos, con ayudas específicas para colectivos concretos, como las personas con discapacidad o las familias numerosas. Las becas se ejecutan en el marco de un determinado sistema educativo, con una distribución dada de competencias entre el Estado y el resto de las administraciones públicas, y bajo un conjunto de normas que rigen todos los aspectos relacionados con su otorgamiento y gestión (Tiana, 2008).

En este sentido, el sistema de becas en España tienen como eje el artículo 27 de la Constitución, que establece el derecho a la educación; la Ley Orgánica de Educación (LOE, 2006), que otorga el derecho de los estudiantes con condiciones desfavorables a obtener becas y ayudas al estudio y por último, el Real Decreto $1721 / 2007$, que establece la regulación básica de las becas y ayudas al estudio territorializadas y no territorializadas. Este Real Decreto, que sustituye al Real Decreto 2298/1983, introduce la posibilidad de que las comunidades autónomas puedan gestionar el sistema estatal de adjudicación de becas. Asimismo, si bien las propias comunidades autónomas han ido desarrollando programas propios de becas y ayudas al estudio que gestionan de forma autónoma, para el período analizado (2004-2005) el Ministerio de Educación gestionaba y administraba aún la mayoría de las becas otorgadas en España (55\% de las becas y $72 \%$ de las cantidades otorgadas).

$4 \quad$ En el caso de las becas, además de las circunstancias socioeconómicas, se establecen unos criterios académicos mínimos para poder gozar de las mismas. 
Cada beca se compone de diferentes elementos que son objeto de agregación en el caso que el solicitante cumpla los requisitos para ser beneficiario de los mismos. Entre los elementos más destacados se encuentra el componente de compensación, el destinado a cubrir los gastos de desplazamiento, el de residencia y el dedicado a sufragar el material didáctico. Además existen otros programas de becas dirigidos a alumnos con necesidades educativas especiales.

Entre los criterios principales para la obtención de las becas destacan los relacionados con el rendimiento académico y con el nivel de renta. Respecto de los requisitos académicos que se exigen para tener derecho a beca, con carácter general, se refieren a la necesidad de matricularse de una cantidad de créditos o módulos y de haber superado una determinada carga lectiva. En cuanto al nivel de renta, se tiene en cuenta la renta familiar que se calcula por agregación de las rentas de cada uno de los miembros de la familia que obtengan ingresos de cualquier naturaleza. El programa de becas fija un umbral de renta no superable para estar en condiciones de solicitarlo, el que se relaciona directamente con el número de miembros del hogar, corregido por el valor catastral de las fincas urbanas que pertenecen a la familia (excluida la vivienda habitual) y por el volumen de actividad económica desarrollada por los miembros de la familia. En la medida que crece el nivel de renta de la familia del solicitante, se van sobrepasando umbrales de renta y, por lo tanto, se van reduciendo los componentes de las becas a los que se tiene derecho. Es importante consignar que en el primer año del nivel secundario postobligatorio solo se exige el cumplimiento de los requisitos económicos, siendo los criterios académicos incorporados en el segundo año.

A nivel de información agregada, y según el Ministerio de Educación, para el período bajo estudio habían 211.770 alumnos becados que recibían 365.745 becas por un importe total de 215.947.200 euros. Los mismos constituían el 16\% del total del alumnado y recibían, a nivel agregado, una cantidad media de 1.019 euros anuales. En cuanto a la distribución geográfica de las becas, la mayoría eran distribuidas en Andalucía (30\%) seguida de la Comunidad Valenciana $(8,6 \%)$ y Canarias $(7 \%)$. Con relación a la tipología de los alumnos becados, $54 \%$ eran mujeres (lo que denota una distribución equitativa de las mismas en lo que se refiere al género); $76 \%$ asistía a centros públicos y la gran mayoría estaba cursando estudios de bachillerato (44\%).

Con el objetivo de describir el contexto en el que se desarrolla la política de becas, se hace una breve referencia a dos indicadores relevantes: el gasto público educativo como porcentaje del PIB y la distribución del mismo según niveles educativos, que incluye la participación que tienen las becas 5 .

Para el año 2008, el 4,62\% del PIB era asignado a gasto público educativo por parte del conjunto de las administraciones públicas y $0,81 \%$ del PIB al gasto de las familias en concepto de pagos de los hogares por servicios educativos. En el contexto internacional, España se situaba por debajo de la media europea (UE-27: 5,07\%), con una evolución apenas favorable desde el año 2000.

Respecto de la distribución del gasto por nivel educativo, para 2007, mientras el 69,4\% correspondía a la educación no universitaria, el 16,6\% a la universitaria, $7,5 \%$ a gasto no distribuido por actividad y el 3,3\% a la formación ocupacional,

5 Fuente: EUROSTAT y Ministerio de Educación (Gobierno de España). 
las becas representaban el 3,2\%. Si bien este porcentaje es relativamente bajo con relación a la comparación internacional (UE-27: 6,4\% en 2008), se debe tener presente que el modelo español se caracteriza por una elevada subvención de los costes (que reduce la matrícula y, por ende, disminuye el monto de la beca destinado a su cobertura). Aún así, queda claro que el objeto de esta investigación corresponde a una política pública educativa que es claramente deficitaria en la comparación internacional con los países del entorno europeo.

\section{Metodología Aplicada para la Evaluación de Políticas Públicas}

La evaluación de políticas públicas es un área de investigación aplicada cuyo objetivo fundamental es valorar la utilidad y el impacto de la intervención pública, teniendo en cuenta que en un entorno de escasez de recursos resulta fundamental evaluar, de forma rigurosa, la actuación que lleva a cabo el sector público, tanto desde la vertiente de la eficiencia en la asignación de los recursos de los que dispone como de la efectividad de las políticas que desarrolla.

En este marco, la evaluación ex post de políticas públicas permite extraer conclusiones para la elaboración de futuras políticas, ya que facilita la detección de errores y, por tanto, introducir mejoras en la racionalización del proceso de toma de decisiones. Se busca contestar cuál habría sido la situación de los beneficiarios si no hubieran participado en el programa. Se trata de una pregunta aplicable a cualquier tipo de intervención y su estudio permite analizar los efectos esperados y no esperados que la intervención genera en los beneficiarios.

Esta idea se puede representar mediante la siguiente ecuación:

$$
\alpha_{i}=Y_{1 i}-Y_{0 i}
$$

donde $Y_{1 i}$ es el rendimiento educativo que el individuo alcanzó cuando participó en el programa; $Y_{0 i}$ representa el rendimiento educativo que el individuo hubiera alcanzado en ausencia del programa; y $\alpha_{i}$ el impacto del programa en la persona " $i$ ". El impacto nunca puede ser observado directamente, siendo conocida esta cuestión como el "Problema Fundamental de Evaluación", debido a que solo una de las dos situaciones potenciales (participar o no participar) es observada para cada individuo en un momento dado (Rubin, 1974).

Así, la evaluación de impacto contará solo con observaciones de $\mathrm{Y}_{1 \mathrm{i}}$ para los individuos beneficiarios del programa, y el problema que se debe solucionar es la estimación de $Y_{0 i}$, el que es llamado comúnmente escenario contrafactual. De acuerdo con Cook y Campbell (1979), el impacto se debe estimar mediante una comparación sistemática entre grupos de población que reciben los beneficios de la intervención (grupos de tratamiento) y grupos de no beneficiarios (grupos de control) con características muy similares en los aspectos más relevantes a los tratados antes de recibir el tratamiento. En este caso, el grupo de control simularía el escenario contrafactual $Y_{0 i}$. Con el objeto de conocer y aislar el efecto neto de una política, se comparan dos grupos: el grupo experimental al que se le ha aplicado el programa y el grupo de control que, estando en iguales condiciones para recibirlo, no lo recibe. Los efectos netos son el resultado de la diferencia de comportamiento entre ambos grupos sobre la variable objetivo considerada. 
La construcción del grupo de control requiere especial atención. El mejor grupo de comparación posible es aquel que representa una imagen fiel del grupo de tratamiento en sus principales características, es decir, respecto de las variables escogidas para determinar sus resultados, pero también en cuanto a los factores no observables como la motivación u otros elementos que afectan el comportamiento del individuo. Se pueden utilizar distintas metodologías de comparación (matching) para construir el grupo de control. Una de ellas es el método del Propensity score matching, ya que permite escoger aquellos individuos que tienen la misma probabilidad o propensión para solicitar la ayuda a partir de una serie de características propias y del entorno de la persona (Caliendo y Kopeining, 2008). Mediante este método de emparejamiento se calcula el efecto medio del tratamiento en los tratados (Average Treatment effect on the Treated o ATT), que determina el valor medio del tratamiento para las personas que recibieron el tratamiento en comparación con los no tratados en el caso hipotético de que ellos también hubieran recibido el tratamiento.

La elección de esta metodología de evaluación se fundamenta en que la misma se ajusta a los requisitos impuestos a partir de la elección del programa a evaluar (programa de becas a nivel español), de la pregunta a resolver (efecto aislado del impacto medio diferencial de las becas según grupo poblacional) y de la naturaleza de los datos disponibles (base de datos individuales longitudinales). Asimismo, esta metodología permite disminuir los efectos de la endogeneidad y no requiere la asunción de una forma funcional determinada.

\section{Análisis Descriptivo}

El análisis se realiza a partir de los datos correspondientes a la Encuesta de Condiciones de Vida (ECV) ${ }^{6}$, elaborada por EUROSTAT con datos longitudinales para el período 2004-2006, publicada en 2009. Los datos disponibles hacen referencia a los países de la Unión Europea y, en el caso español, la muestra agrupada comprende 58.740 individuos. La componente longitudinal permite seguir en el tiempo a las mismas personas, estudiar los cambios que se producen en sus vidas cuando las condiciones y las políticas socioeconómicas se modifican, y cómo reaccionan a estos cambios.

La población de referencia son los hogares y todas las personas mayores de 16 años que se encuentren residiendo en un hogar dentro del territorio de los estados miembros en el momento de realizarse la encuesta. La base de datos proporciona información personalizada sobre ingresos, educación, salud, ocupación, entre otros, que permite conocer las condiciones en que viven los encuestados y las posibles situaciones de pobreza y exclusión social. En el caso de los ingresos, es de especial interés para este trabajo la información relacionada con las transferencias en dinero recibidas por el individuo por concepto de becas y, en el caso de las variables educativas, aquellas que permiten seguir su evolución dentro del sistema educativo. Aquí es importante subrayar que la

6 Contrato n. EU-SILC/2006/19. En él se establece la obligación, en el momento de publicar los resultados, de comunicar lo siguiente: "EUROSTAT no es responsable de los resultados y las conclusiones, responsabilidad que corresponde al investigador". 
evaluación realizada se limitará al impacto de las becas que suponen un ingreso monetario para el beneficiario, debido a que no se dispone de información de las prestaciones en especie (becas de matrícula, por ejemplo).

Para el estudio de impacto de las becas y ayudas al estudio en el rendimiento educativo de los estudiantes, la variable dependiente hace referencia al nivel educativo que posee la persona a los 19 años. Si bien la edad teórica para finalizar el nivel secundario postobligatorio son los 18 años, se ha optado por seleccionar un año más para evitar encontrar individuos con 18 años que aún no tengan este nivel educativo alcanzado solo porque la encuesta se ha realizado antes de finalizar su curso lectivo. Como variables independientes se consideran todas las variables con información disponible relacionadas con el individuo, sus progenitores, su hogar y el entorno que lo rodea. Seguidamente, se describen la totalidad de las variables empleadas para el análisis empírico (véase Tabla 1).

\section{TABLA 1}

VARIABLES UTILIZADAS EN EL ANÁLISIS EMPÍRICO

\begin{tabular}{|c|c|c|}
\hline Tipo de variable & & Descripción \\
\hline \multirow[t]{6}{*}{ Individuo } & $\begin{array}{l}\text { Nivel educativo a los } \\
19 \text { años }\end{array}$ & $\begin{array}{l}\text { Variable que hace referencia al nivel educativo que } \\
\text { posee el individuo a la edad de } 19 \text { años en } 2006 . \\
\text { Se estructura como una dummy con valor = } 1 \text { si la } \\
\text { persona tiene un nivel educativo igual o superior } \\
\text { al de secundaria postobligatoria (ISCED-97) }{ }^{1} \\
\text { (Post_oblig_con_19). }\end{array}$ \\
\hline & Beca & $\begin{array}{l}\text { Variable dummy que indica si el individuo ha recibido } \\
\text { una o más becas o ayudas al estudio durante el período } \\
\text { 2004-2005 (Beca). }\end{array}$ \\
\hline & Género & $\begin{array}{l}\text { Dummy género. Toma el valor } 1 \text { si el individuo es } \\
\text { mujer (Mujer). }\end{array}$ \\
\hline & "Efecto calendario" & $\begin{array}{l}\text { Dummy mes de nacimiento. Toma el valor } 1 \text { si } \\
\text { el individuo nació en el último trimestre del año } \\
\text { (Último_tri). }\end{array}$ \\
\hline & Estado de salud & $\begin{array}{l}\text { Dummy enfermedad crónica. Toma el valor } 1 \text { si el } \\
\text { individuo padece una enfermedad o incapacidad } \\
\text { crónica (Enf_crónica). }\end{array}$ \\
\hline & Orden entre hermanos & $\begin{array}{l}\text { Variable que hace referencia al orden que ocupa el } \\
\text { individuo con relación a sus hermanos (ejemplo: el } \\
\text { hermano mayor tiene un número de orden igual a } \\
\text { 1) (Posición). }\end{array}$ \\
\hline \multirow[t]{3}{*}{ Padre/Madre } & Nivel educativo padre & $\begin{array}{l}\text { Máxima educación lograda por el padre (ISCED-97) } \\
\text { (Educ_padre). }\end{array}$ \\
\hline & $\begin{array}{l}\text { Nivel educativo } \\
\text { madre }\end{array}$ & $\begin{array}{l}\text { Máxima educación lograda por la madre (ISCED-97) } \\
\text { (Educ_madre). }\end{array}$ \\
\hline & Ocupación padre & $\begin{array}{l}\text { Variable que indica la clase social del padre, que se } \\
\text { elabora a partir del ISCO- } 88 \text { (Ocup_padre })^{2} \text {. }\end{array}$ \\
\hline
\end{tabular}


Tabla 1 (cont.)

\begin{tabular}{|c|c|c|}
\hline \multirow[t]{4}{*}{ Tipo de variable } & & Descripción \\
\hline & Ocupación madre & $\begin{array}{l}\text { Variable que indica la clase social de la madre, que } \\
\text { se elabora a partir del ISCO- } 88 \text { (Ocup_madre). }\end{array}$ \\
\hline & Actividad padre & $\begin{array}{l}\text { Dummy activo. Toma el valor } 1 \text { si el individuo se } \\
\text { encuentra activo (Activo_padre). }\end{array}$ \\
\hline & Actividad madre & $\begin{array}{l}\text { Dummy activo. Toma el valor } 1 \text { si el individuo se } \\
\text { encuentra activo (Activo_madre). }\end{array}$ \\
\hline \multirow[t]{8}{*}{ Hogar } & Tamaño del hogar & $\begin{array}{l}\text { Variable que hace referencia al número total de } \\
\text { miembros del hogar (Tamaño_hogar). }\end{array}$ \\
\hline & Número de hermanos & $\begin{array}{l}\text { Variable que indica la cantidad de hermanos existentes } \\
\text { en el hogar (Nro_hermanos). }\end{array}$ \\
\hline & Nivel de ingresos (I) & Quintil de ingresos disponibles equivalentes (Quintil) ${ }^{3}$. \\
\hline & Nivel de ingresos (II) & $\begin{array}{l}\text { Dummy dificultades económicas. Toma el valor } 1 \\
\text { si el hogar declara tener problemas para asumir los } \\
\text { gastos habituales del mes (Dificultad_econ). }\end{array}$ \\
\hline & $\begin{array}{l}\text { Régimen de la } \\
\text { vivienda }\end{array}$ & $\begin{array}{l}\text { Dummy propietario de la vivienda. Toma el valor } \\
1 \text { si los habitantes del hogar son propietarios de la } \\
\text { misma (Vivienda_prop). }\end{array}$ \\
\hline & $\begin{array}{l}\text { Problemas } \\
\text { estructurales }\end{array}$ & $\begin{array}{l}\text { Dummy problemas estructurales en la vivienda. } \\
\text { Toma el valor } 1 \text { si existen problemas estructurales } \\
\text { en la vivienda (Prob_estructural). }\end{array}$ \\
\hline & $\begin{array}{l}\text { Dimensiones del } \\
\text { hogar }\end{array}$ & $\begin{array}{l}\text { Dummy más de cuatro dependencias en el hogar. } \\
\text { Toma el valor } 1 \text { si el hogar tiene más de cuatro } \\
\text { dependencias (Mas_4_dep) }{ }^{4} \text {. }\end{array}$ \\
\hline & $\begin{array}{l}\text { Grado de } \\
\text { urbanización }\end{array}$ & $\begin{array}{l}\text { Dummy si el individuo vive en una zona de baja o } \\
\text { media urbanización. (Baja_media_urb). }\end{array}$ \\
\hline
\end{tabular}

Fuente: Elaboración propia a partir de microdatos de EUSILC LONGITUDINAL UDB 2006 versión 2 - de marzo de 2009.

1 ISCED-97: International Standard Classification of Education.

2 ISCO-88: Internacional Standard Classification of Occupations. Este índice se basa en la clasificación presentada por Erikson/Golfthorpe/Portocarero, que permite categorizar a las familias en diferentes clases sociales a partir de sus ocupaciones (Erikson et al., 1979).

3 El ingreso equivalente se calcula teniendo en cuenta el ingreso disponible anual del hogar, el factor de no respuesta y el tamaño equivalente del hogar, el que pondera de manera diferencial a los adultos y a los menores del hogar (escala: OCDE modificada).

4 La variable utilizada incorpora habitaciones, salas para comer, salas de estar y altillos o sótanos habitables. Asimismo, incorpora la cocina solo en el caso que cumpla además con una de las funciones antes descritas. Quedan excluidos el baño, la despensa, los pasillos y la cocina en caso que solo se emplee para cocinar.

A partir del total de observaciones válidas para la variable dependiente (Post_oblig_con_19), se genera una subbase de datos con 783 observaciones de individuos de 19 años durante el período analizado que contiene valores perdidos para algunas de las variables independientes, básicamente localizadas en aquellas que hacen referencia a los progenitores. Para sustituir los valores perdidos, se ha empleado la técnica de imputación múltiple (Schafer, 1999; 
TABLA 2

ANÁLISIS DESCRIPTIVO (N=783)

\begin{tabular}{|c|c|c|c|c|}
\hline & Media & $\begin{array}{l}\text { Desviación } \\
\text { estándar }\end{array}$ & Mínimo & Máximo \\
\hline \multicolumn{5}{|l|}{ Variable dependiente } \\
\hline Post_oblig_con_19 & 0,45 & 0,49 & 0 & 1 \\
\hline \multicolumn{5}{|c|}{ Variables independientes } \\
\hline \multicolumn{5}{|l|}{ Individual } \\
\hline Beca & 0,19 & 0,39 & 0 & 1 \\
\hline Mujer & 0,48 & 0,49 & 0 & 1 \\
\hline Último_tri & 0,26 & 0,44 & 0 & 1 \\
\hline Enf_crónica & 0,07 & 0,26 & 0 & 1 \\
\hline Posición & 1,57 & 0,70 & 1 & 3 \\
\hline \multicolumn{5}{|l|}{ Padre / madre } \\
\hline Educ_padre & 2,47 & 1,51 & 1 & 5 \\
\hline Educ_madre & 2,28 & 1,38 & 1 & 5 \\
\hline Ocup_padre & 3,69 & 1,08 & 1 & 5 \\
\hline Ocup_madre & 3,28 & 1,54 & 1 & 5 \\
\hline Activo_padre & 0,89 & 0,32 & 0 & 1 \\
\hline Activo_madre & 0,52 & 0,50 & 0 & 1 \\
\hline \multicolumn{5}{|l|}{ Hogar } \\
\hline Tamaño_hogar & 4,17 & 0,99 & 1 & 6 \\
\hline Nro_hermanos & 1,32 & 0,88 & 0 & 3 \\
\hline Quintil & 2,63 & 1,37 & 1 & 5 \\
\hline Dificultad_econ & 0,63 & 0,48 & 0 & 1 \\
\hline Vivienda_prop & 0,83 & 0,37 & 0 & 1 \\
\hline Prob_estructural & 0,17 & 0,38 & 0 & 1 \\
\hline Mas_4_dep & 0,71 & 0,45 & 0 & 1 \\
\hline Baja_media_urb & 0,54 & 0,49 & 0 & 1 \\
\hline
\end{tabular}

Fuente: Elaboración propia a partir de microdatos de EUSILC LONGITUDINAL UDB 2006 versión 2 - de Marzo 2009.

Acock, 2005). Esta técnica, de característica estocástica, permite hacer un uso eficiente de los datos, obtener estimadores no sesgados y reflejar la incertidumbre que la no respuesta parcial introduce en la estimación de los parámetros (Rubin, 1996). Su aplicación se basa en sustituir los datos no observados por $\mathrm{m}>1$ valores posibles simulados.

Una vez obtenida la base de datos completa, se realiza un primer análisis descriptivo de las variables que integran el análisis empírico (véase Tabla 2). En cuanto a los resultados más destacables referentes a las variables individuales, se observa que el porcentaje total de alumnos que tiene el nivel secundario postobligatorio aprobado con 19 años es del $45 \%^{7}$. Asimismo, el 19\% de los mismos reconocían haber recibido una beca en algún momento dentro del período analizado. Si bien no es del todo comparable, este valor se considera esperable a partir de los datos publicados por el Consejo Escolar del Estado que indican

7 Como referencia, en el caso de la Encuesta de Población Activa 2006 (II Trimestre), el porcentaje era de $49,57 \%$. 
una media de $18 \%$ para los alumnos de ciclos formativos de grado medio y de $16 \%$ para los alumnos de bachillerato, para el período evaluado.

Con relación a las variables referentes a los progenitores, solo una variable denota una diferencia sustancial entre padres y madres. La misma hace referencia a la tasa de actividad de unos y otros, con una clara preeminencia del género masculino. Finalmente, los principales resultados a partir del análisis descriptivo de las variables del hogar muestran que el tamaño medio es de cuatro miembros, donde $63 \%$ dice tener dificultades económicas y $83 \%$ posee la vivienda donde habita en régimen de propiedad. En cuanto a la distribución espacial de los hogares, existe una distribución equitativa entre los hogares urbanos y aquellos ubicados en zonas periféricas y/o en el ámbito rural.

\section{Resultados Empíricos}

\subsection{Efecto total "neto" de la becas}

La evaluación del impacto "neto" de las becas se realiza mediante una técnica de emparejamiento cuasiexperimental: el Propensity score matching. Esta metodología permite determinar el impacto de una política y su estimación está compuesta por dos etapas.

En primer lugar, se realiza un análisis de máxima verosimilitud (logit) para estimar las probabilidades de cada individuo de obtener una beca en el nivel secundario postobligatorio, a partir de todas las variables individuales y del entorno. Como resultado de la estimación (véase Anexo 1), existen cuatro elementos que estarían determinando la posibilidad de recibir una beca en la secundaria postobligatoria: la posición entre los hermanos, la situación económica (quintil y tenencia en propiedad de la vivienda) y, finalmente, el lugar de residencia.

La significatividad de la posición se explica en que son los hijos mayores de los hogares los que tienen más posibilidades de continuar sus estudios más allá de la escolarización obligatoria y, por tanto, los que pueden solicitar una beca. Asimismo, la importancia de los aspectos económicos era esperada debido a los requisitos legales para la obtención de una beca en España y tiene una correlación con los datos oficiales que indican una mayor concentración de las becas en Andalucía, región donde un importante porcentaje de su población vive bajo condiciones socioeconómicas muy limitadas. En cuanto a la variable referida a la residencia, queda clara la prioridad que tienen los alumnos que habitan en áreas consideradas de mediana o baja urbanización. Este elemento también tiene una marcada correlación con el puntaje adicional otorgado en la normativa a los alumnos provenientes de entornos rurales. Finalmente, destacar que el género no resulta significativo. Tal resultado se condice con la legislación vigente que no discrimina según esta variable y con los resultados oficiales que indican una paridad de género entre los alumnos beneficiarios de las becas.

Como conclusión, este resultado global, si bien herramental para la aplicación de la metodología de evaluación, en sí mismo es relevante, porque permite afirmar que los resultados siguen, en cierta forma, los requisitos legales para el otorgamiento de una beca y va en línea con lo publicado para España por la Agencia de Evaluación y Calidad (AEVAL, 2009).

En segundo lugar, y para conocer el efecto diferencial de haber recibido una beca en personas con igual probabilidad de obtenerla (ATT), se han desarro- 
llado diversos métodos de emparejamiento, siendo cuatro los más conocidos y que se aplicarán en la presente evaluación: método del Vecino más Próximo, de Estratificación, Kernel y Radius (Becker e Ichino, 2002). Los resultados (véase Tabla 3) muestran la efectividad de las becas y ayudas al estudio otorgadas debido a su efecto positivo $($ ATT $>0)$ para las diferentes metodologías de cálculo aplicadas. En todas las estimaciones, el efecto medio del tratamiento es significativamente diferente de cero, con lo que se puede afirmar que existe un efecto "neto" positivo de las becas y ayudas al estudio sobre el nivel educativo logrado por los individuos de 19 años. Si tenemos en cuenta que la variable objetivo varía entre 0 y 1 , los coeficientes obtenidos muestran que las becas incrementan entre $22,2 \%$ y $25,7 \%$ las probabilidades de acabar con éxito el nivel secundario postobligatorio al comparar dos individuos similares en sus características observables.

TABLA 3

IMPACTO “NETO” DE RECIBIR UNA BECA EN EL RENDIMIENTO EDUCATIVO

\begin{tabular}{|c|c|c|c|c|c|}
\hline & & \multicolumn{2}{|c|}{ Estadístico t } & \multicolumn{2}{|c|}{ Individuos } \\
\hline & ATT & Analítico & Bootstrapping & Tratados & Control \\
\hline Vecino M.P.(a) & $0,234^{\mathrm{a}}$ & 5,063 & (d) & 150 & $527-528$ \\
\hline Error estándar & & 0,046 & & & \\
\hline Vecino M.P.(b) & $0,235^{\mathrm{a}}$ & 5,085 & (d) & 150 & $527-528$ \\
\hline Error estándar & & 0,046 & & & \\
\hline Estratificación & $0,257^{\mathrm{a}}$ & 5,838 & 6,002 & 150 & 621 \\
\hline Error estándar & & 0,044 & 0,043 & & \\
\hline Kernel (c) & $0,243^{a}$ & - & 5,580 & 150 & 621 \\
\hline Error estándar & & & 0,044 & & \\
\hline Radius & $0,222^{a}$ & 4,630 & 4,529 & 150 & $527-550$ \\
\hline Error estándar & & 0,048 & 0,049 & & \\
\hline
\end{tabular}

a Significativa al $99 \%$.

Todas las estimaciones se han realizado con reemplazo y soporte común. (a) Vecino más próximo con selección aleatoria. (b) Vecino más próximo con ponderativos idénticos. (c) En el caso de la aproximación Kernel mediante la función de densidad Epanechnikov-Kernel el resultado es de 0,251. (d) No se calculan siguiendo a Abadie e Imbens (2008).

Fuente: Elaboración propia a partir de microdatos de EUSILC LONGITUDINAL UDB 2006 versión 2 - de marzo de 2009.

\subsection{Efectos diferenciales}

En esta parte del análisis, y central para la investigación, se trata de establecer la existencia de efectos diferenciales mediante la estimación del impacto medio del tratamiento (ATT) en el caso de diferentes submuestras determinadas. Para cada una de ellas se realiza un proceso independiente de estimación como el aplicado anteriormente para el total de individuos. Consecuentemente, se determinan diferentes valores del parámetro de impacto según la técnica de comparación aplicada y según la base de datos imputada que se emplee. Para 


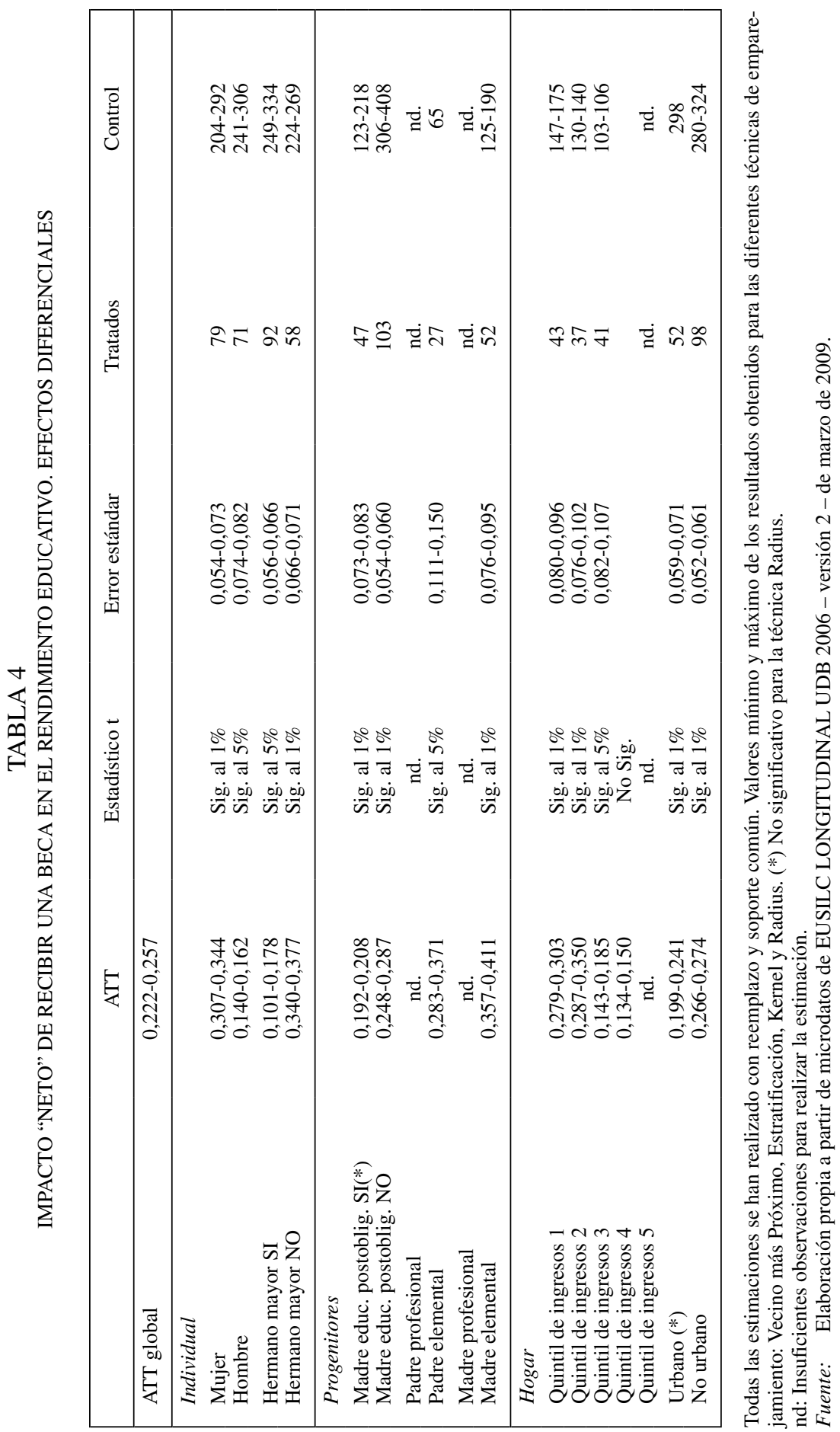


una fácil visualización de los mismos, se ha escogido presentar los resultados como un rango entre los valores mínimos y máximos estimados y solo se citan las variables con efectos diferenciales significativos respecto del parámetro medio global (véase Tabla 4).

En cuanto a la interpretación de los resultados, se debe resaltar la existencia de efectos diferenciales provocados por algunas de las características observables de los individuos y su entorno. A nivel individual, existe un efecto positivo que refuerza el efecto medio global en el caso del género femenino y otro efecto, en este caso negativo, que penaliza a los hermanos mayores. Claramente, ser mujer o no ser el hermano mayor en el hogar son características personales que influyen en un mayor aprovechamiento de la beca percibida en términos de rendimiento académico. En ambos casos, un colectivo consigue triplicar el efecto de una misma beca que su contraparte.

Del ámbito de los progenitores, los resultados muestran que los becados cuyas madres tienen un menor nivel educativo y ocupan empleos de menor cualificación estarían aprovechando en mayor grado la ayuda pública, hecho que se vería reflejado en un incremento proporcionalmente mayor de las tasas de éxito escolar en el nivel secundario postobligatorio.

En referencia al hogar, se han encontrado resultados significativos en el caso de individuos provenientes de hogares pertenecientes al quintil 1 y 2 , que refuerza el impacto de las becas con relación al valor medio obtenido para toda la población. Es importante observar cómo el efecto de las becas es creciente en la medida que desciende el nivel económico de las familias. Por último, no existe un resultado del todo concluyente sobre la existencia de un efecto diferencial a partir del lugar de residencia.

\subsection{Calidad de los emparejamientos}

Con el objeto de comprobar la calidad de los emparejamientos realizados, seguidamente se presentan los resultados obtenidos del análisis de diferencias de medias entre el grupo tratado y el grupo de control efectivamente empleado. Asimismo, se presenta un análisis gráfico para evaluar la conveniencia de la imposición del soporte común en la estimación.

\subsubsection{Análisis de diferencias de medias}

Una de las opciones que plantea la literatura, con el objeto de conocer si la aplicación de la metodología cuasiexperimental ha permitido generar dos grupos de observación similares, es el análisis de las diferencias de medias entre el grupo tratado y el grupo de control. En caso de obtener un resultado satisfactorio, se podría asegurar que los parámetros de impacto estimados realmente estarían detectando la diferencia existente en la variable resultado solo como consecuencia del tratamiento realizado.

Para corroborar la relativa similitud entre ambos grupos se emplea un test t de diferencias de medias. Los resultados obtenidos indican que, de las 16 variables incorporadas en el análisis, siete muestran diferencias significativas, ocho presentan diferencias no significativas y una, la referida a la ocupación del padre, no ofrece un resultado concluyente (véase Tabla 5). 


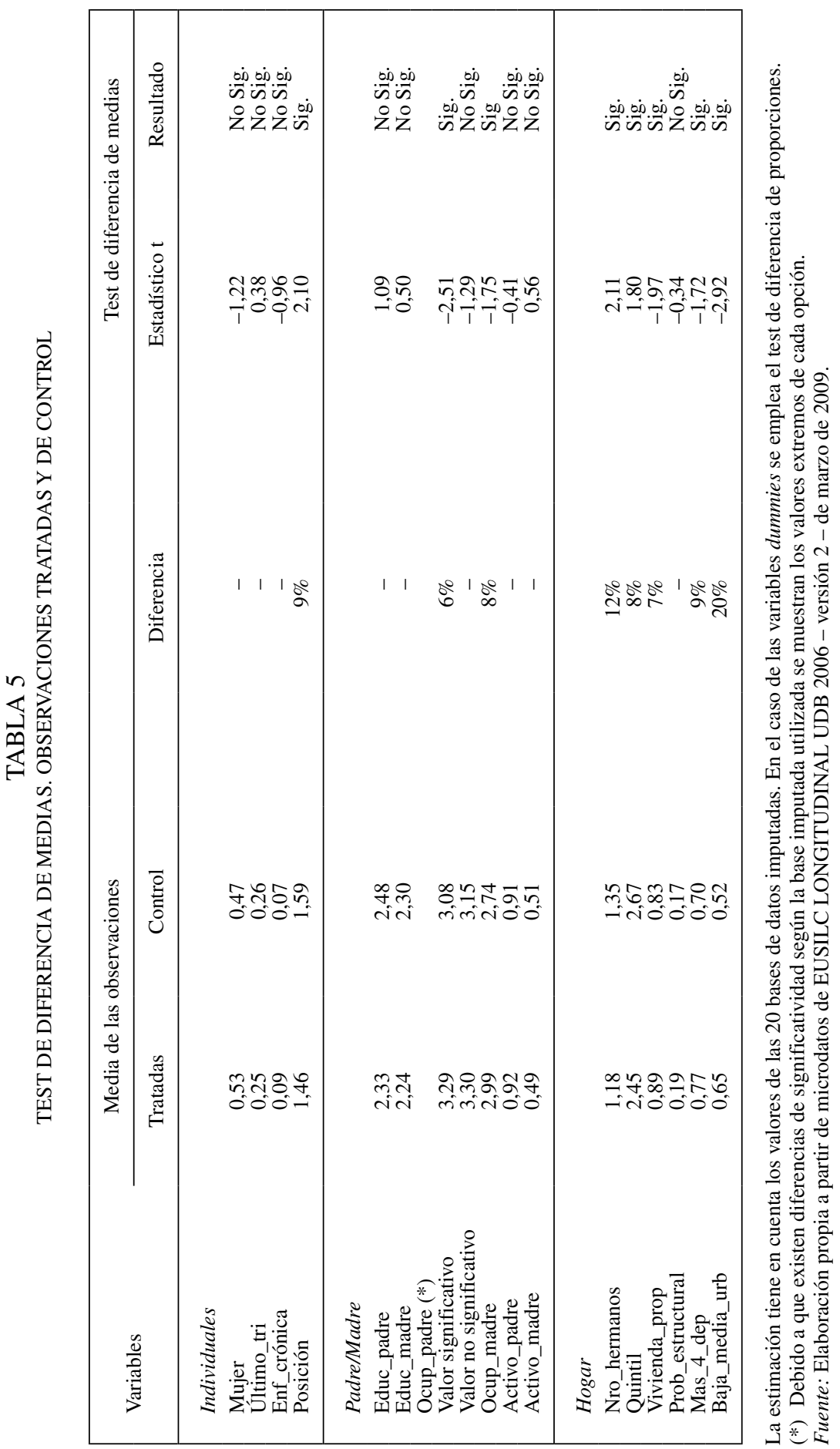


Seguidamente, se analiza en detalle aquellas variables con diferencias significativas en sus valores medios. En este sentido, se observa que en seis de las siete variables que presentan diferencias estadísticamente significativas las mismas no resultan relevantes en sus valores absolutos. En este colectivo se ubicarían las variables referidas a la posición del individuo, la ocupación de la madre, el número de hermanos, el quintil de ingresos, la tenencia en propiedad de la vivienda y la referida al tamaño del hogar. En segundo lugar, solo la variable referida a la ubicación del hogar se presenta con una diferencia significativa y relevante en sus medias (20\%). Por tanto, y a partir del comportamiento agregado de la totalidad de las variables tenidas en cuenta, se puede concluir que el análisis de comparación se realiza a partir de dos grupos similares y, por tanto, que el efecto en el rendimiento educativo encontrado proviene realmente de la posibilidad de haber gozado de algún programa de becas durante el período analizado (2004-2006).

\subsubsection{Comprobación de la conveniencia de la imposición del soporte común}

Para comprobar que la aplicación del soporte común es una precondición válida para realizar las estimaciones, se realiza un análisis visual mediante un gráfico que muestra las funciones de densidad Kernel del Propensity Score para el caso de las observaciones tratadas y de control (véase Gráfico 1). Se observa

\section{GRÁFICO 1 \\ DENSIDAD DE KERNEL PARA LAS OBSERVACIONES TRATADAS Y DE CONTROL}

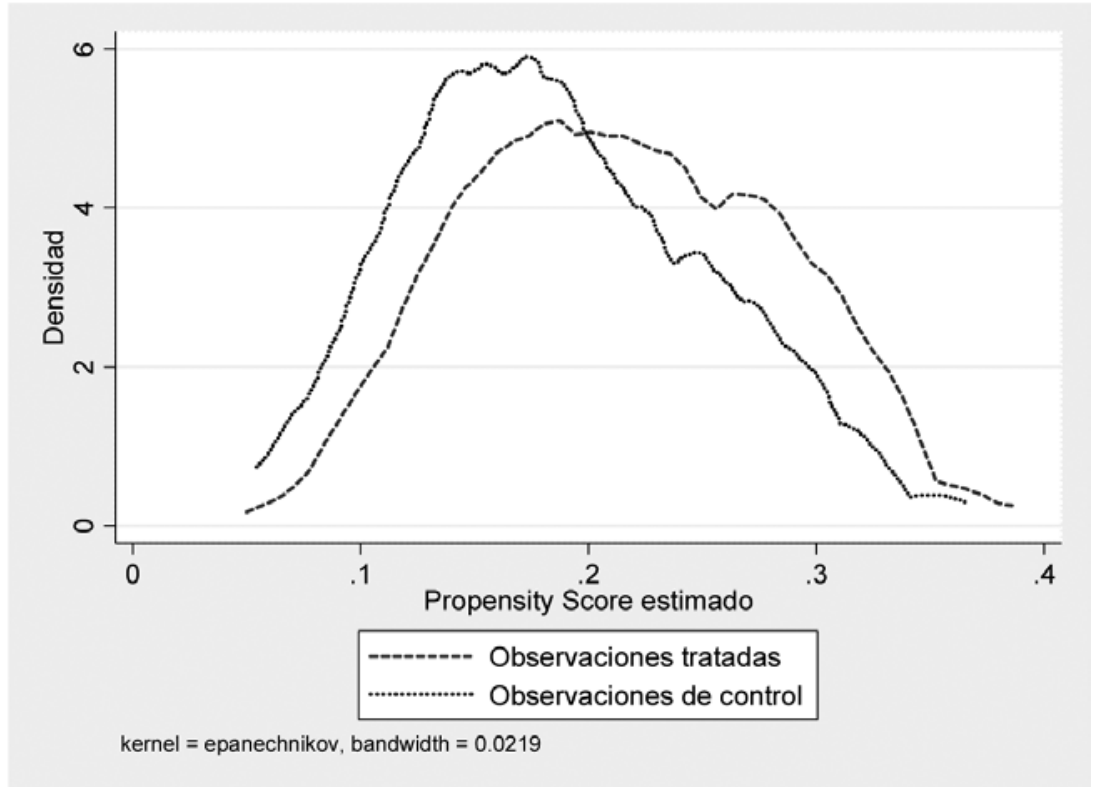

Fuente: Elaboración propia a partir de microdatos de EUSILC LONGITUDINAL UDB 2006 versión 2 - de marzo de 2009. 
que para casi la totalidad de los Propensity Scores estimados existe una observación tratada y una de control. Esto sugiere que el supuesto de soporte común permite mejorar la calidad de los emparejamientos sin perder observaciones relevantes, ya que el 98,47\% de la base original queda incluida dentro del área empleada para la estimación.

\subsection{Evaluación agregada del impacto}

La literatura identifica dos principales efectos que se generan como consecuencia de la aplicación de una política pública: los directos, sobre los individuos participantes, y los efectos indirectos, que producen una amplificación de los mismos hacia los no participantes (Heckman et al., 1999). Por tanto, idealmente la estimación de los mismos debería realizarse mediante un enfoque de equilibrio general, el que requiere de un alto nivel de sofisticación en la modelación de las futuras interacciones entre los agentes económicos.

En el presente estudio se ha optado por un análisis de equilibrio parcial a partir de suponer que la base de datos empleada (longitudinal) y la metodología aplicada (PSM) permiten aislar el efecto de la política de otros efectos contemporáneos y, además, que los efectos indirectos de las mismas son relativamente pequeños con relación a los efectos directos en los beneficiarios. Es evidente que en el cumplimiento de ambos supuestos radica una clara limitación a la metodología empleada existiendo la posibilidad de sobrestimar los efectos de la política bajo análisis.

Con el objeto de intentar introducir un análisis agregado que complemente el análisis realizado de la política de becas, seguidamente se realiza una evaluación económica mediante un análisis de coste-efectividad. Este método se caracteriza por utilizar como unidad de medida los resultados obtenidos como consecuencia de la implementación de una política determinada. En cuanto a la medición de los costes de implementación, la literatura propone dos opciones de cálculo. En primer lugar, la metodología del bottom-up donde se detallan y cuantifican todos los elementos generadores de costes. Para ello, el investigador debe poseer una vasta y rica información. En segundo lugar la opción del top-down, donde el coste total es dividido por la totalidad de beneficiarios. Debido a las limitaciones informativas existentes, se opta por esta segunda opción a la hora de calcular los costes de implementación de la política de becas en España.

El resultado del análisis cuasiexperimental antes descrito determinaba un efecto neto de la política de entre $22,2 \%$ y $25,7 \%$ de incremento en la tasa de finalización del nivel educativo analizado. Para traducir este porcentaje a una cantidad de alumnos que indique la efectividad del programa, se emplea la información oficial publicada en el Instituto Nacional de Estadística (http://www. ine.es) para el curso 2004/2005. Si bien la información con el grado de detalle requerido solo está disponible para los alumnos matriculados en Bachillerato, ya que es la titulación principal en el nivel secundario postobligatorio en España, se sigue con el análisis teniendo en cuenta que los resultados obtenidos podrían extrapolarse al resto de titulaciones existentes.

En el curso de 2004/2005 habían 613.581 alumnos matriculados en Bachillerato, cuya duración es de dos años, de los cuales 216.281 alumnos van a promocionar del primero al segundo año y 201.361 alumnos van a terminar el segundo año 
con la consecuente graduación. Por tanto, el resto de los alumnos (195.939) conformaba el grupo considerado de no éxito, sea porque no acabó el primero o el segundo año de Bachillerato. Si bien los datos no son comparables, se debe destacar que el $32 \%$ del total de matriculados que representa este último grupo tiene una estrecha relación con las tasas de abandono escolar prematuro publicadas para esos años por el EUROSTAT (32\% en 2004 y 30,8\% en 2005).

En el análisis, la efectividad del programa estaría vinculada a la potencialidad de las becas de incrementar el colectivo de alumnos que continúan o finalizan el nivel secundario postobligatorio en España. En este sentido, y con los datos del Bachillerato, se puede estimar un efecto concreto de la política pública en términos de cantidad de alumnos retenidos por el sistema educativo. La aproximación cuasiexperimental indicaba un incremento en las posibilidades de éxito escolar en términos de retención escolar de $22 / 25 \%$. Por tanto, si se incrementase la tasa agregada real de éxito escolar observada en el curso 2004/2005 (68\%) en el porcentaje indicado por la estimación cuasiexperimental se llegarían a tasas del 83/85\%. Como consecuencia directa de este crecimiento, se observaría una reducción en el colectivo de no éxito hasta llegar a cifras del 15/17\%, magnitudes mucho más cercanas al objetivo planteado por la Unión Europea para 2020 (10\%). En términos absolutos, la aplicación de la política de becas de forma generalizada permitiría que 91.630 alumnos continúen escolarizados en este nivel no obligatorio del sistema educativo español.

En cuanto a los costes de la política, los mismos se calculan teniendo en cuenta la inversión agregada total realizada por las diferentes administraciones públicas españolas en becas y ayudas al estudio para el curso 2004/2005 en alumnos de Bachillerato. El monto total del gasto público por estos conceptos ascendía a 75,6 millones de euros. Por tanto, la ratio coste/resultado de la política ascendería a 825 euros (75.632.000/91.630), indicando el coste por unidad de resultado obtenido.

A nivel de análisis comparado, resulta relevante comprobar que el coste unitario necesario para que un alumno de Bachillerato sea retenido por el sistema educativo es inferior al importe medio de las becas en España para el curso lectivo analizado (1.019 euros). Esto permite intuir, aunque no confirmar, un mayor aprovechamiento de la ayuda pública por parte de los estudiantes de Bachillerato con relación al resto de colectivos de estudiantes de otras titulaciones pertenecientes al mismo nivel educativo.

\section{Conclusiones}

La presente investigación tiene su motivación en dos elementos: las altas tasas de abandono escolar prematuro que presenta España y la manifiesta necesidad de impulsar estudios y análisis que intenten evaluar la efectividad real de las políticas públicas y, entre ellas, las políticas educativas.

El objetivo principal del trabajo es analizar el efecto de las becas y ayudas al estudio en las tasas de éxito escolar en el nivel secundario postobligatorio en España. Por ello, y a partir de la base de datos longitudinal publicada por EUROSTAT en 2009, se evalúa el impacto diferencial (por subgrupos poblacionales) de dichas ayudas públicas en el rendimiento educativo. Con ese objetivo, 
se emplea una metodología ampliamente utilizada en otros ámbitos pero escasamente aplicada en España para la evaluación de las políticas educativas, un diseño cuasiexperimental mediante el Propensity score matching.

La interpretación de los resultados indica que las becas incrementan en más de $20 \%$ las posibilidades de finalizar con éxito el nivel secundario postobligatorio con la edad teórica de finalización, para el caso de dos individuos con similares características. Asimismo, los resultados obtenidos permiten aseverar la presencia de efectos diferenciales en el impacto de la política según algunas características propias del individuo, de sus progenitores y del hogar donde habita el mismo. Se confirma la existencia de un efecto diferencial positivo en el caso de las mujeres y de los alumnos provenientes de hogares desfavorecidos. Además, se determina la existencia de otros elementos adicionales que también resultan significativos a la hora de generar efectos diferenciales, como la educación y empleo de los progenitores. Adicionalmente, se comprueba la existencia de correlación entre los requisitos básicos que legalmente se requieren para que un alumno pueda recibir una beca, los datos publicados por el Ministerio de Educación y los resultados obtenidos en la estimación. Por último, y de manera complementaria, el análisis de coste-efectividad permite observar que la inversión media requerida para mejorar las tasas de retención en el Bachillerato sería más baja que la inversión necesaria en el resto de titulaciones correspondientes al nivel secundario postobligatorio.

En términos de implicaciones sobre la política educativa, el presente trabajo aporta una nueva evidencia sobre el rol dinamizador del rendimiento educativo que proporcionan las becas escolares en el nivel secundario postobligatorio en España. Asimismo, se demuestra que el efecto de la misma no se distribuye de forma homogénea entre los perceptores de las ayudas y, por tanto, el propio diseño de las políticas de becas puede mejorar la efectividad de las mismas en términos de éxito escolar, en un contexto de altas tasas de abandono escolar prematuro en España. En este sentido, siendo conscientes de la gran diversidad de aptitudes y necesidades de nuestros alumnos, cuesta entender la actual homogeneidad y falta de flexibilidad existente en la normativa vigente. Por tanto, un diseño de la política de becas más ajustado a las características propias y del entorno del individuo permitiría incrementar la eficacia del gasto público, entendido como el efecto de la misma en las tasas de finalización lograda por el alumno beneficiado.

Finalmente, las limitaciones del trabajo se han originado, principalmente, en las propias de la base de datos utilizada debido a la existencia de información solo sobre aquellas ayudas públicas, en concepto de becas y ayudas al estudio, que significaron un ingreso monetario para el individuo. Finalmente, las escasas observaciones han impedido poder realizar un análisis comparativo por CC.AA.

\section{Bibliografía}

Abadie, A. e Imbens, G. (2008). "On the failure of the bootstrap for matching estimators", Econometrica, Vol. 76; 1537-1557.

Acock, A. (2005). "Working with missing values", Journal of Marriage and Family, Vol. 67, 1012-1028. 
Adame Obrador, M. y Salvà Mut, F. (2010). "Abandono escolar prematuro y transición a la vida activa en una economía turística: el caso de Baleares”, Revista de Educación, Vol. 51, 185-210.

AEVAL (Agencia Estatal de Evaluación de las Políticas Públicas y la Calidad de los Servicios). (2009). Evaluación del sistema general de becas educativas. Primera evaluación: Diagnóstico de la situación actual y principales alternativas para mejorar su eficacia. Madrid.

Alegre Canosa, M. y Benito Pérez, R. (2010). "Los factores del abandono educativo temprano. España en el marco europeo", Revista de Educación, Número extraordinario, 65-92.

Barham, V., Boadway, R., Marchand, M y Pestieau, P. (1995). "Education and the poverty trap", European Economic Review, 39, 1257-1275.

Becker, S. e Ichino, A. (2002). "Estimation of average treatment effects based on propensity scores", The Stata Journal, Vol. 2, 358-377.

Berti Ceroni, C. (2001). "Poverty Traps and Human Capital Accumulation", Economica, 68, 203-219.

Caliendo, M. y Kopeinig, S. (2008). "Some Practical Guidance for the Implementation of Propensity Score Matching", Journal of Economic Surveys, Vol. 22, 31-72.

Cameron, L. (2009). "Can a public scholarship program successfully reduce school drop outs in a time of economic crisis? Evidence from Indonesia", Economics of Education Review, 29, 308-317.

Cardoso, E. y Portela Souza, A. (2004). "The impact of cash transfers on child labor and school attendance in Brazil", Working Papers $N^{\circ}$ 04-W07. Vanderbilt University, en http://www.hec.unil.ch/ocadot/SECODEVdocs/ Articles/Cardoso-Souza.pdf

Cook, T. y Campbell, D. (1979), "Quasi-experimentation. Desing \& analysis issues for field settings". Boston: Houghton Miffin Company.

De Pablos Escobar, L. y Gil Izquierdo, M. (2008). "Análisis de la incidencia de reformas en el sistema de financiación de la educación universitaria en España a partir de un modelo de comportamiento", Hacienda Pública Española, Vol. 184, 117-152.

Deke, J. (2003). "A study of the impact of public school spending on post-secondary educational attainment using statewide school district refinancing in Kansas", Economics of Education Review, Vol. 22, 275-284.

DesJardins, S., Ahlburg, D. y McCall, B. (2002). "Simulating the longitudinal effects of changes in financial aid on student departure from college", The Journal of Human Resources, Vol. 37, 653-679.

Dronkers, J. (2008). "Education as the backbone of inequality - European education policy: constraints and possibilities". En F. Becker; K. Duffek y T. Mörschel (eds.), Social Democracy and Education. The European Experience (51-135). Amsterdam: Friederich Ebert Stiftung / Karl Renner Institut / Wiardi Beckman Stichting.

Escudero Muñoz, J. M., González González, M. T. y Martínez Domínguez, B. (2009). "El fracaso escolar como exclusión educativa: comprensión, políticas y prácticas", Revista Iberoamericana de Educación, Vol. 50, 41-64.

Erikson, R., Goldthorpe, J. y Portocarero, L. (1979). "Intergenerational class mobility in three Western European Societes: England, France and Sweden", British Journal of Sociology, Vol. 30, 415-441. 
European Commission (2010). "Europe 2020. A European strategy for smart, sustainable and inclusive growth". Brussels, Communication from the Commission, en http://ec.europa.eu/eu2020/pdf/

GHK. (2005). "Study on access to education and training, basic skills and early school leavers". Brussels: European Commission.

Heckman, J, Lalonde, R. y Smith, J. (1999). "The economics and econometrics of active labor market programs," en Handbook of Labor Economics. A. Ashenfelter and D. Card eds: Elsevier Science, pp. 1865-2097.

Katz, L. y Murphy, K. (1992). "Changes in relative wages, 1963-1987: Supply and demand factors", The Quarterly Journal of Economics, Vol. 107, 35-78.

Kim, D. (2007). "The effect of loans on students' degree attainment: Differences by student and institutional characteristics", Harvard Educational Review, Vol. 77, 64-100.

Marcenario Gutiérrez, O y Navarro Gómez, L. (2001). "Un análisis microeconómico de la demanda de educación superior en España", Estudios de economía aplicada, Vol. 19, 69-86.

McPherson, M. y Schapiro, M. (1991). "Does student aid affect college enrollment? New evidence on a persistent controversy", The American Economic Review, Vol. 81, 309-318.

Monks, J. (2009). "The impact of merit-based financial aid on college enrollment: A field experiment", Economics of Education Review, Vol. 28, 99-106.

OCDE. (1998). Human Capital Investment. Paris: OCDE.

Roca Cobo, E. (2010). "El abandono temprano de la educación y la formación en España”, Revista de Educación, Número extraordinario, 31-62.

Rubin, D. (1974). "Estimating causal effects of treatments in randomized and non-randomized studies", Journal of Educational Psychology, Vol. 66, 688-701.

Rubin, D. (1996). "Multiple imputation after 18+ Years", Journal of the American Statistical Association, Vol. 91, 473-489.

Rumberger, R. (1987). "High school dropouts: A review of issues and evidence", Review of Educational Research, Vol. 57, 101-121

Schafer, J. (1999). "Multiple imputation: a primer", Statistical Methods in Medical Research, Vol. 8, 3-15.

Skoufias, E. (2001). "PROGRESA and its impacts on the human capital and welfare of households in rural Mexico: A synthesis of the results of an evaluation by IFPRI", Research Report. International Food Policy Research Institute, Washington, en http://www.ifpri.org/sites/default/ files/publications/skoufias_finalsyn.pdf

Tiana, A. (2008). "La política de becas del Estado: un balance del período 20042008”, Presupuesto y Gasto Público, Vol. 50, 191-200.

Wetzel, J., O'Toole, D. y Peterson, S. (1999). "Factors affecting student retention probabilities: A case study", Journal of Economics and Finance, Vol. 23, 45-55. 


\section{Anexo}

TABLA A1

DETERMINANTES DE RECIBIR UNA BECA (logit)

\begin{tabular}{|c|c|c|c|c|}
\hline Variable dependiente: Beca $(y=0,1)$ & Coeficiente & $\begin{array}{c}\text { Error } \\
\text { estándar }\end{array}$ & Estadístico t & $P$-value \\
\hline \multirow{2}{*}{\multicolumn{5}{|c|}{$\begin{array}{l}\text { Variables independientes } \\
\text { Individual }\end{array}$}} \\
\hline & & & & \\
\hline Mujer & 0,211 & 0,197 & 1,07 & 0,283 \\
\hline Último_tri & $-0,092$ & 0,227 & $-0,41$ & 0,683 \\
\hline Enf_crónica & 0,344 & 0,353 & 0,98 & 0,329 \\
\hline Posición & $-0,310^{\mathrm{b}}$ & 0,154 & $-2,00$ & 0,046 \\
\hline \multicolumn{5}{|l|}{ Padre/Madre } \\
\hline Educ_madre & 0,107 & 0,091 & 1,18 & 0,239 \\
\hline Ocup_padre & 0,126 & 0,088 & 1,41 & 0,159 \\
\hline Ocup_madre & 0,056 & 0,077 & 0,73 & 0,466 \\
\hline Activo_padre & 0,312 & 0,355 & 0,88 & 0,380 \\
\hline Activo_madre & $-0,104$ & 0,206 & $-0,50$ & 0,615 \\
\hline \multicolumn{5}{|l|}{ Hogar } \\
\hline Quintil & $-0,142^{b}$ & 0,075 & $-2,03$ & 0,036 \\
\hline Dificultad_econ & 0,370 & 0,245 & 1,51 & 0,131 \\
\hline Vivienda_prop & $0,744^{\mathrm{a}}$ & 0,319 & 2,34 & 0,019 \\
\hline Prob_estructural & 0,055 & 0,262 & 0,21 & 0,833 \\
\hline Mas_4_dep & 0,333 & 0,235 & 1,42 & 0,157 \\
\hline Baja_media_urb & $0,435^{b}$ & 0,217 & 2,00 & 0,045 \\
\hline $\mathrm{N}^{\circ}$ Observaciones & 783 & & & \\
\hline Log-Likelihood & $-364,27$ & & & \\
\hline LR Chi2 (16) & 36,44 & & & \\
\hline $\mathrm{P}>\mathrm{Chi} 2$ & 0,0476 & & & \\
\hline
\end{tabular}

a Significativo al $99 \%$.

b Significativo al $95 \%$.

Para la estimación se han excluido las variables referidas al tamaño del hogar y al número de hermanos debido a que se encuentran contempladas en la variable quintil de ingreso. Asimismo, se ha quitado de la estimación la variable referida a la educación del padre por su alta correlación con la educación de la madre. Se ha optado por escoger la variable que presentaba un mayor número de observaciones válidas.

Fuente: Elaboración propia a partir de microdatos de EUSILC LONGITUDINAL UDB 2006 versión 2 - de marzo de 2009. 